\title{
THE PREDATORY BEHAVIOR OF SOME ARANEID SPIDERS AND THE ORIGIN OF IMMOBILIZATION WRAPPING*
}

\author{
By Michael H. Robinson, Heath Mirick and Olga Turner \\ Smithsonian Tropical Research Institute, P.O. Box 2072, \\ Balboa, Canal Zone (Panama)
}

The evolution of predatory behavior in web building spiders is a subject of considerable interest. Functionally the spider has to solve two problems immediately after prey strikes the web. It has to locate the prey and then to attack it in such a way that the prey is prevented from escaping and is subdued. The spider may then be confronted with further problems involved in freeing the prey from the web and in transporting it to a feeding or storage site. Spiders may attack solely by biting, may bite some types of prey and wrap others in silk, or may rely entirely on silk as an attack weapon. Silk may also be used, after the initial attack, at other stages in the process of prey capture. Eberhard (1967) has reviewed the possible stages in the evolution of the use of silk for attack. By comparing the uses of silk in the predatory behavior of several representatives of a number of families of web building spiders he arrived at the conclusion that immobilization by wrapping in silk is derived from post immobilization wrapping. We fully agree with his conclusions but feel that a comprehensive treatment of the subject requires a more detailed consideration of the uses of silk by araneid spiders. Araneids may use the wrapping process at four different (and in all probability, functionally distinct) stages in the prey capture sequence. Furthermore, the behavioral components of the wrapping process are not necessarily common to all four cases. If these facts are considered, the picture of the evolution of wrapping behavior by web building spiders becomes more complex than Eberhard (ibid) assumed.

This paper examines data on the predatory use of silk by Nephila clavipes (Linnaeus), Argiope argentata (Fabricius), A. savingnyi Levi and $A$. florida Chamberlin \& Ivie, as well as other araneid spiders. In addition, we report on an experimental investigation into the functional efficiency of two basic attack strategies used by these spiders. We finally propose a scheme by which complex predatory repertoires can be derived from simpler ones by an essentially additive process.

*Manuscript received by the editor August 22, 1969 


\section{Terminology}

Confusion can arise over the use of the terms immobilization and post immobilization. This is because the word immobile is frequently regarded as synonymous with motionless. This problem of terminology is a difficult one. Robinson ( 1969) used the expression restraint wrapping in preference to immobilization wrapping since restraint does not have connotations of motionlessness. However, if restraint is used as a word to qualify biting attacks, two separate effects may be confounded. Thus the bite may effect restraint simply because the prey is held in the chelicerae, whereas the addition of poison, by means of the bite, ensures a degree of immobilization after the spider releases its hold. In the interests of consistency we have decided to refer to wrapping used as a means of initial restraint as immobilization wrapping and to use "post immobilization wrapping" to designate all the forms of wrapping that occur after an initial atack by biting. This usage is not meant to imply that immobilization wrapping renders the prey absolutely motionless, or that post immobilization wrapping is applied to completely motionless prey. Immobilization wrapping does seem to effectively immobilize prey by preventing those forms of movement which could result in escape from the web. Similarly the immobilization bite does not necessarily render the prey motionless but does reduce the level of movements to a stage at which the spider can safely stop biting. Eberhard (1967, p. I77) seems to recognize this distinction without explicitly stating it. Thus, in describing the behavior of diguetid spiders, he refers to the (immobilization) bite being maintained "until the prey's struggles subsided", but also states that the prey were "usually still twitching" when the bite ceased.

\section{Wrapping by Nephila clavipes}

Data on the behavior of Nephila clavipes are derived from a recent study by Robinson \& Mirick (in prep.). Nephila clavipes bites all prey and does not enswathe prey in silk prior to biting. Wrapping occurs in three sets of circumstances:

I. Wrapping occurs at the feeding site (the hub of the web) and is there applied to prey which have previously been treated in two different ways. All prey small enough to be pulled from the web at the capture site and carried to the hub in the jaws are wrapped on arrival at the hub. All prey which have been wrapped at the capture site (after biting) and subsequently carried to the hub in the jaws, are rewrapped at the hub. This is post immobilization wrapping at the feeding site. 
2. Wrapping occurs at the capture site if the prey cannot be pulled from the web after the immobilization bite. Such prey is wrapped, cut from the web, and then transported to the hub. This is post immobilization wrapping at the capture site, type $\mathrm{I}$.

3. Wrapping occurs at the capture site after the prey has been freed from the web by pulling, if it is too large to be carried in the jaws. It is then carried, suspended on silk, from leg IV. This is post immobilization wrapping at the capture site, type 2.

Wrapping by Argiope species

The predatory behavior of Argiope argentata has recently been described by Robinson ( I969), and further analysis is given by Robinson \& Olazarri (in press). Details of the predatory behavior of $A$. savignyi and $A$. florida are essentially similar (Robinson \& Robinson, in prep.).

All the above species of Argiope, and many other araneids, use silk as an attack weapon, prior to biting, and in this respect their predatory behavior differs fundamentally from that of Nephila clavipes. The Argiope species also wrap prey in silk in other circumstances:

I. Prey which have been seized in the jaws and pulled from the web are subsequently wrapped at the hub (see Nephila I.). Prey wrapped at the capture site but transported to the hub in the jaws are also wrapped at the hub. This is post immobilization wrapping at the feeding site.

2. Prey which have been immobilized by biting are wrapped at the capture site following the long bite. The greater proportion of lepidopteran prey is treated in this way. This is post immobilization wrapping at the capture site.

3. Prey which are carried in the jaws and become tangled in the web during transportation are wrapped and carried on silk. This is post immobilization wrapping during transportation.

Immobilization wrapping, post immobilization wrapping at the capture site, and post immobilization wrapping during transportation also occur in the case of Araneus diadematus (Peters I93I, I933a, 1933b).

Two forms of immobilization wrapping occur in $A$. argentata, $A$. savignyi and $A$. florida. These differ in the initial behavioral component which has been called throwing (Robinson 1969). In this behavior the spider throws skeins of silk over the prey, using both 
legs IV to direct the throw. In one form of immobilization wrapping the spider is in contact with the prey whilst throwing, whereas in the more complex form it throws swathes of silk over the prey from a distance. In an as yet unidentified species of Eriophora that we have studied, the spider actually turns to face away from the prey during non-contact throwing.

The behavior of Argiope argentata following the wrap/short bite couplet is important to our consideration of the functional aspects of immobilization wrapping. After the wrap/short bite sequence the spider leaves the prey in situ and returns to the hub. Eventually, after a variable period of time, it returns to the prey, cuts it out of the web and transports it to the hub. We believe that this period during which the spider leaves the prey and returns to active monitoring of the web has important implications for the functional interpretation of immobilization wrapping.

After the long bite/wrap sequence the spider does not leave the prey in situ but proceeds to cut it from the web and carry it to the hub. If, however, there is already previously caught prey at the hub, the spider leaves the newly caught prey at the capture site and returns to feed on the previously caught prey. Peters (I93I) notes that Araneus diadematus also leaves prey in situ if it already has prey at the hub.

\section{Functional considerations}

We have so far described araneids with one and two basic methods of attack (biting alone and biting or wrapping). In both cases there are distinct forms of employment of post immobilization wrapping. Eberhard (1967) has argued that post immobilization wrapping at the feeding site may have been the first type of wrapping behavior to appear, followed by post immobilization wrapping at the capture site. Immobilization wrapping could then be derived from post immobilization wrapping at the capture site. In terms of function Eberhard (ibid, p. 180) comments "wrapping may have originated as a postimmobilization process designed to free the spider for subsequent attacks".

It certainly seems reasonable to assume that post immobilization wrapping at the feeding site would ensure that the spider could make further attacks without dropping, or otherwise losing, the previously caught prey. (In fact, we have evidence that when Nephila clavipes omits this behavior, under the pressure of a rapid succession of prey, it can lose prey that it has already caught as it rushes to attack new prey; see page 497). Post immobilization wrapping at the capture 
site is another matter. In the case of Nephila it does not immediately free the spider to make new attacks. After such wrapping Nephila carries the prey to the hub and never leaves it in situ. Post immobilization wrapping at the capture site, by Nephila, must, therefore, have other functions. It certainly reinforces the immobilization achieved by the biting attack, as must post immobilization wrapping at the feeding site. There are, however, good grounds for assuming that this is not the primary function. Capture site wrapping, type I, can be elicited by manipulating the prey, experimentally, in such a way that it cannot be pulled from the web by the spider. It is then a response to a failure of the prey removal process (Robinson \& Mirick, in prep.). It seems reasonable to suppose that such capture site wrapping functions primarily to allow the spider to cut the prey from the web without losing it. The prey package resulting from the wrapping process is compact, partially disentangled from the viscid spiral, and securely attached to a radial web member. Eberhard (1967, p. I77) reported an instance in which a diguetid spider anchored a large prey to the web (with silk) before releasing it from the chelicerae and removing it from the web. This seems to be a functionally similar process to the capture site wrapping, type I, by Nephila.

When wrapping occurs at the capture site, after the prey has been freed from the web by pulling (type 2), the function seems to be to facilitate transportation to the hub. After such wrapping the prey is carried suspended from leg IV, and not in the jaws. Both Argiope argentata and Araneus diadematus carry prey from below the hub, and above a certain weight range, on silk rather than in the jaws (Robinson 1969, Peters 1933b). In these spiders, and Argiope savignyi, A. florida and Eriophora sp., this carrying technique occurs after post immobilization wrapping at the capture site, type I. All the spiders that we have studied, i.e., the Argiope spp., Nephila clavipes, and Eriophora sp., build webs which are inclined to the perpendicular to a greater or lesser extent. The spider rests on the undersurface of the web and almost always carries its prey along that surface, or close to it. The lower portion of the web (below the hub) is normally greater in area than the upper portion. When carrying prey from the lower portion the spider is walking against the slope. This means that prey carried in the jaws, and therefore partially beneath the spider, are in a position which potentially favors entanglement during transportation. Prey carried beneath the spider, on silk, hang away from the web and are thus much less likely to become entangled during transportation. The 
position is reversed in the upper part of the web. There, prey carried on silk would tend to hang awkwardly against the spider's body or legs. Argiope argentata does not carry prey from the upper part of the web on silk. Prey of a weight which would be carried on silk in the lower web are often carried in the jaws from the upper web. Very heavy prey, in the upper web, are moved to the hub, after wrapping, by a complex process which has been called the "derrick technique" (Robinson \& Olazarri, in press). Thus the role of post immobilization wrapping, at the capture site, in facilitating transportation, is probably most clearly illustrated by consideration of activities in the lower part of the web. Transportation of prey with long and projecting appendages must inevitably be facilitated by the "trussing" effect of wrapping, irrespective of the means of transportation.

Once post immobilization wrapping at the capture site, type I, has evolved it may be exploited for a further function. Prey can then be safely left in situ and the process of removal from the web, and transportation, can be deferred until later. If the spider already has prey at the hub it may be advantageous to return from attacks as quickly as possible, and only cut out and carry further prey when it is ready to feed upon them. This process would split up a single sequence into two shorter sub-sequences. Nephila does not do this but transports all prey and stores them at the hub. All the Argiope species that we have studied store prey, in situ, in the web. (Storing prey in the web may increase the danger that they will be stolen by theridiid kleptoparasites. These are frequently found in association with the webs of Nephila clavipes and Argiope argentata in Panama. The very large size of Nephila webs may increase the spider's difficulties in detecting the activities of the kleptoparasites and this could account for the fact that Nephila does not store prey in the web.)

The step from post immobilization wrapping at the capture site to attack wrapping must confer adaptive advantages. It seems improbable that these are related to the immediacy of escape prevention since the three species of Argiope described above, and Araneus diadematus, retain the immobilization bite for precisely those prey that have rapid escape potentials. (After Robinson, 1969, reported the use of the immobilization bite for non vibrating lepidopterans, as well as lepidopterans in general, we have found that this is true for several other, as yet undetermined, species of tropical araneids.) Where attack wrapping involves the prey being trapped under layers of silk thrown over it from a distance, it may enable spiders to attack prey with a diminished risk of injury to themselves. Certainly we have seen a Nephila lose a portion of its leg to the biting mouthparts 
of a katydid during a close quarters attack. In fact, Nephila attacks large prey with legs I and II raised off the web and flexed back, and often darts forward to make a short bite and then backs off before attacking again. A succession of short bites and retreats may thus occur.

Wrapping attacks may be initiated while the spider is actually standing on the prey. These may still protect the spider from injury since it can maintain a greater distance between itself and the prey than is possible during the more intimate bite. We feel, however, that immobilization wrapping may confer a further and very important advantage. It may help to achieve an economy of time spent away from the hub while attacking and immobilizing prey, as Robinson (1969) suggested. Any reduction in the time spent in capturing prey must enable the spider to be in a better position to respond to further prey before they can escape from the web. Additionally, if the spider is more vulnerable to predators when it is attacking prey, it would be advantageous to reduce time spent out on the web.

There is a statistically significant difference between the duration of the bite given before wrapping (in the long bite/wrap sequence) and the duration of the bite given after wrapping (in the wrap/short bite sequence). The long bite may be long simply because the spider has to wait for it to take effect before it can safely release the prey and commence wrapping. In the case of immobilization wrapping, the prey is secured before the bite is given, and the spider gives a short bite and retires to the hub to resume "monitoring" the web. The short bite may inject a smaller venom dose, or one which is slower acting. However, since the prey is wrapped, it can safely be left while the venom takes effect. The effects on living prey of the two types of bite are under investigation. There is certainly a difference in the time spent at the capture site when the spider uses the two types of attack against the same type of prey (Robinson, 1969, p. I 70 ).

The above conclusions about function can be summarized as follows:

I. Post immobilization wrapping at the feeding site results in the prey being safely anchored to the web by silk. It functions to permit the spider to make further attacks without losing the prey "in hand". In effect, the spider does not have to lose time securing the prey to the web at the moment when a new prey strikes the web (i.e., when it needs to be able to make an immediate attack). That this is a correct interpretation is suggested by the fact that those prey which are carried suspended on a silk thread are not wrapped 
at the hub, but merely attached by the transport thread, on arrival. Thus the transport thread functions as an attachment thread. Wrapped prey that are carried in the jaws do not have a transport thread and are wrapped again at the hub.

2. Post immobilization wrapping at the capture site reinforces the immobilization achieved by the poisoning bite, facilitates the removal of strongly adhering prey from the web, and enables the spider to safely store prey in situ. In addition, it may facilitate the transportation of large or heavy prey. The behavior of Nephila provides evidence for the separate functions associated with prey removal and transportation. Thus prey which Nephila can remove by pulling are not wrapped in the web but may be wrapped before being carried to the hub. Argiope only pulls very small prey from the web, and cuts out all other prey after wrapping has produced a compact prey package. The occurrence of post immobilization wrapping during transportation is a further example of wrapping functioning to facilitate transportation.

3. Immobilization wrapping effectively immobilizes prey, and compared with immobilization biting, allows the spider to attack without bringing its more vital parts into direct contact with the prey. It may thus be less dangerous than immobilization biting. Furthermore it is economical of time and this may be a very important factor.

As far as we are aware there are no araneids that do not bite after immobilization wrapping. Uloborids, on the other hand, rely exclusively on wrapping for prey immobilization (Eberhard 1967). The short bite of araneids, or at least those that we have studied, contains some poison since prey removed from the prey package after the short bite eventually become completely quiescent. It therefore seems probable that the short bite supplements the effect of the immobilization wrapping. Uloborids are all quite small spiders and it seems possible that total reliance on immobilization wrapping might be less efficient in the case of the araneids (which may rely heavily on stronger and more active prey).

The performance of Argiope argentata and Nephila clavipes confronted with a rapid succession of small prey

In the above discussion of the functional aspects of wrapping behavior we have stressed the possibility that economy of time spent in prey capture sequences may be obtained by leaving prey in situ after it is wrapped. We have also pointed out that this is possible as a consequence of both immobilization wrapping and post immobilization wrapping at the capture site. Economy in time spent away 
Table 1.

A comparison of times spent away from the hub of the web by Argiope argentata and Nephila clavipes with Trigona sp. as prey.

A. Uninterrupted sequence (single Trigona). Prey $\mathrm{n}=50$, Spider $\mathrm{n}=10$ of each sp.

$\begin{array}{ccccc}\text { Mean total } & \text { Range } & \begin{array}{c}\text { Standard } \\ \text { deviation }\end{array} & \begin{array}{c}\text { Standard error } \\ \text { of mean }\end{array} \\ \text { Nephila } & 70 \text { secs. } & 44-108 & 15 & 2.1 \\ \text { Argiope } & 77 \text { secs. } & 38-192 & 40 & 5.6\end{array}$

B. Ten Trigona per spider, arriving at 30 second intervals (see text for explanation). Data from 50 successful sequences of attack by 10 spiders of each species.

$\begin{array}{ccccc} & \begin{array}{c}\text { Mean total } \\ \text { time. }\end{array} & \text { Range } & \begin{array}{c}\text { Standard } \\ \text { deviation }\end{array} & \begin{array}{c}\text { Standard error } \\ \text { of mean }\end{array} \\ \text { Nephila } & 30 \text { secs. } & 14-65 & 11.8 & 1.7 \\ \text { Argiope } & 18.7 \text { secs. } & 7-33 & 7.8 & 1.1\end{array}$

Times in A are for the total prey capture sequence: attack, removal from the web, transportation, storage at the hub. In B the times for Argiope are for attack, storage in the web and the spiders' return to the hub (since the spider omitted removal of the prey from the web and its transportation).

from the hub may be especially critical under conditions when prey arrive in rapid succession. Both Nephila clavipes and Argiope argentata deal with stingless bees (Trigona sp.) in predatory sequences of similar duration, see Table I. However, Argiope uses immobilization wrapping whereas Nephila does not wrap the bees until it reaches the hub. Agriope has the potential of being able to omit the immediate removal of the prey from the web, and its transportation, whereas Nephila has not. We, therefore, decided to test the two spiders under conditions when several prey were arriving at the web in succession. To do this we allowed one bee to fly towards either a Nephila or an Argiope web every thirty seconds until ten prey had flown at the web. The Trigona were released from a glass tube held 18 " from the web and 6" vertically below the spider. By having a light source behind the web we ensured that the bees would fly towards the web rather than in any other direction. The dispersion of the bees on hitting the web was approximately standardized by the constant position of the escape tube. Each of 20 well fed Nephila and 20 well fed Argiope (mature females in each case) were tested with one succession of to bees. We made notes of the fate of each insect and timed and noted the behavior of the spider. The results are shown in Figure I. This reveals a basic difference in the prey 


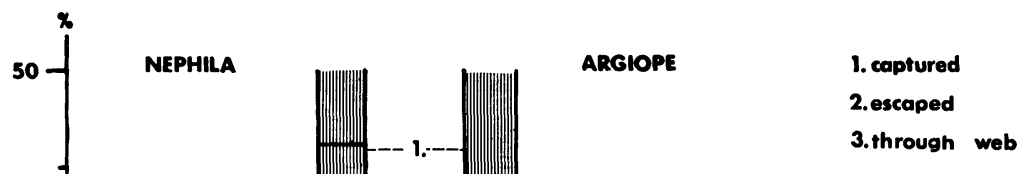

Figure 1. Stepped histograms showing, in a simplified and condensed form, the results of the experiments in which Nephila clavipes and Argiope argentata were presented with a succession of small prey. Details of the experiment are given in the text. The basal histogram (3) of each block shows the percentage of prey passing through the webs. The center histogram (2) shows the percentage escaping after hitting the web, and the behavior of the spider at the time the prey escaped. The top histogram (1) of each block shows the percentage of prey captured (reading from $100 \%$ by subtraction). In the case of Nephila some prey were lost after capture and these are shown in the lower part of the capture column. Number of prey presented to each species: 200 . 
capture efficiency of the two webs for small insects. Thus (basal columns, Figure I), $15.5 \%$ of the Trigona flew straight through the Argiope webs, missing the viscid spiral, or touching the web momentarily. Only $2 \%$ of the Trigona passed through the Nephila webs. Since we started, in all cases, with fresh webs, this result is not likely to be biased by the effects of the prey arriving early in the sequence on the state of the web for later arrivals. In fact, in the Argiope results, escapes through the webs in the first five of the sequence were in the relationship 17:14 compared with escapes in the second five of the sequence.

The second columns in each graph show the number of escapes occurring after the prey were in the web and an analysis of the behavior of the spider at the time the prey escaped. Argiope missed only $24.5 \%$ of the prey striking the web whereas Nephila missed $40.5 \%$. If we add losses through the web to these figures we find that Argiope lost $40 \%$, and Nephila $42.5 \%$ of potential prey flying at the web. In addition, as mentioned earlier, Nephila incurred further losses of captured prey when it rushed into attack carrying these prey in its jaws (having omitted post immobilization wrapping at the feeding site). An additional $3.5 \%$ of prey were lost in this way. The total absolute losses for Nephila were thus $46 \%$.

The fact that $15.5 \%$ of the bees passed through the Argiope web meant that this spider was under reduced pressure during the five minute experimental period compared with Nephila. However, analysis of the data shows that Argiope achieved a very considerable acceleration of predatory behavior under the conditions of the experiment, and became significantly faster than Nephila (see Table I). A substantial part of this reduction was achieved by the omission of the cut out, carry, and wrap at hub stages and is therefore a striking illustration of the advantage of being able to leave immobilized prey in situ. Prey losses by Nephila occurred principally when it was performing activities at the hub (post immobilization wrapping and turning to resume its normal head down position). The attack phase (biting) was usually completed by the time the next prey arrived. A surprising number of escapes occurred while Nephila was locating the prey (i.e., at the pluck stage). These losses may be attributable to the fact that prey location did not occur at the moment of impact but was delayed by preceding activities until the prey had almost freed itself. Argiope was back at the hub, unencumbered by prey, when nearly half of the losses occurred. It seems quite possible that, in this case, the presence of numbers of wrapped prey in the web may complicate further prey location. We also got 


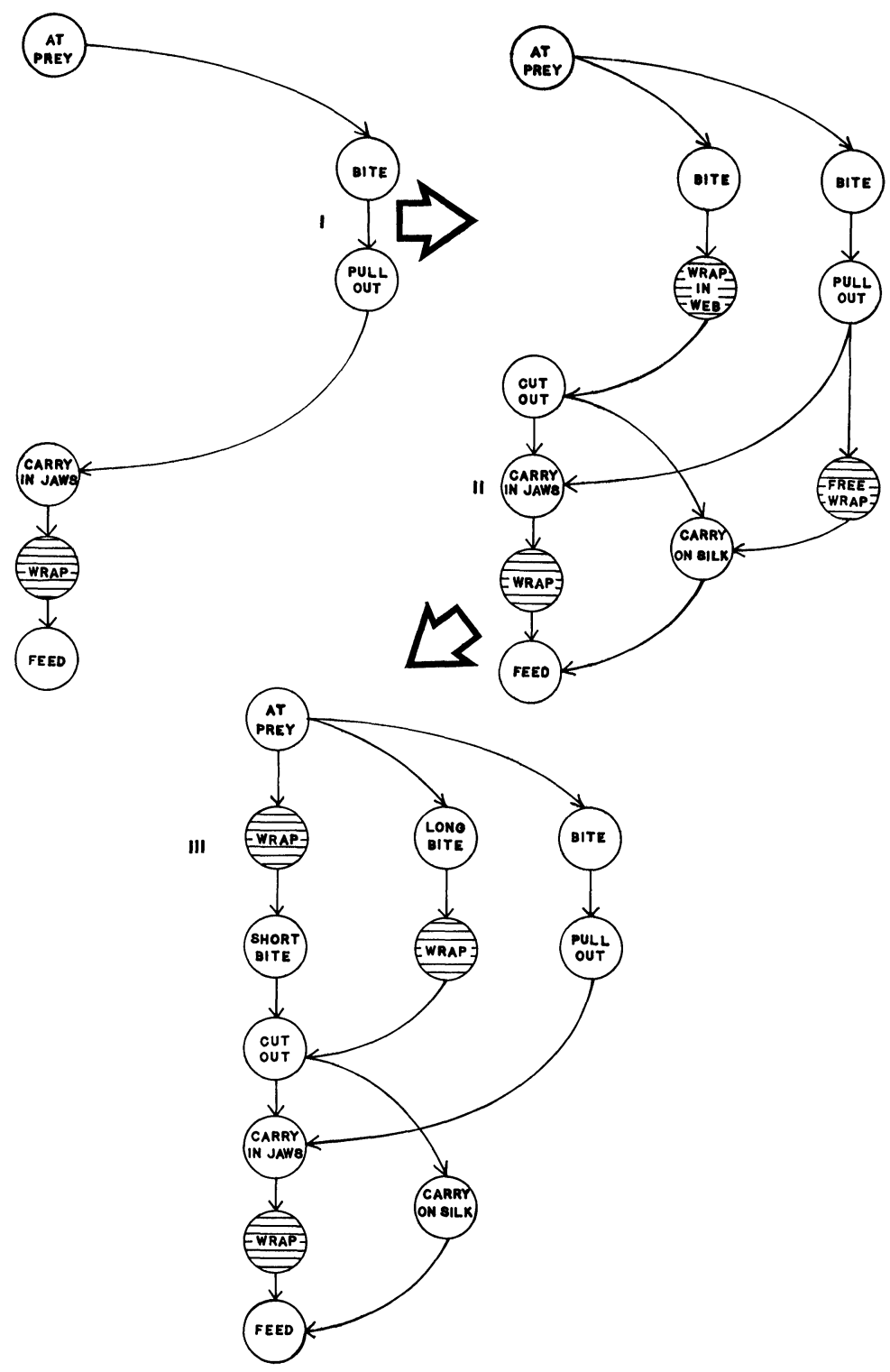


the distinct impression, that, in at least some of these cases, the spider was ignoring newly arrived prey.

In a sense, the conditions of this experiment were purely arbitrary, and may never even be approximated under natural conditions. (We have, however, records of large numbers of social insects being caught in short periods.) The experiment does show that Argiope can compensate for its (for small prey) much less efficient web under certain conditions. We also believe that immobilization wrapping, and the subsequent possibility of quickly leaving the prey in situ is the key to this success.

\section{Conclusion}

We feel that it is possible to reconstruct the possible steps in the evolution of immobilization wrapping by considering the behavior of existing araneid spiders. This process eliminates the necessity to extrapolate from the behavior of spiders of other families which may be very distant from the line of araneid evolution. The explanation we propose below also accounts for the existence of several forms of attack behavior in some of the araneids which have developed attack by wrapping. We offer an adaptive function for each step in the process and envisage the evolution of complex predatory patterns in araneids to have been additive.

We propose the following scheme:

Stage I. All prey overcome by biting. Prey pulled from the web in the jaws and carried to the hub where post immobilization wrapping occurs. This stage is not found in any araneid whose predatory behavior is described but is represented in the behavior of Nephila and Argiope to small prey. Function of wrapping at the hub: to prevent loss of prey during subsequent attacks.

Figure 2. An additive scheme illustrating the stages in the evolution of prey wrapping by araneids as proposed by the authors. The model for each stage is simplified, behavior prior to arrival at the prey is omitted and the spider's capacity to interrupt a sequence before the cut out stage is not shown. The circles represent a behavioral unit and where more than one arrow leaves, or enters, a circle the behavior may be followed, or preceded, by the behaviors indicated. Stage 1 is hypothetical, but occurs as part of Stages $2 \& 3$. Stage 2 represents the prey capture sequences of Nephila clavipes, and with the omission of free wrap is a model of the behavior of some species of Micrathena and Gasteracantha. Stage 3 occurs in Argiope spp. and Eriophora sp., and may be typical of most "advanced" araneids. 
Stage 2. (a) All prey overcome by biting. Post immobilization wrapping occurs at the capture site when the prey cannot be pulled from the web in the jaws. This stage occurs in Nephila clavipes. Function of wrapping enmeshed prey at the capture site: to permit the spider to safely remove its chelicerae from the prey for use in cutting it from the web. All prey stored at the hub.

(b) All prey overcome by biting. In addition to post immobilization wrapping of enmeshed prey there is wrapping of prey freed from the web by pulling. This wrapping occurs before transportation on silk. Function: to permit the safe transportation of prey too large to be carried in the jaws. This stage also occurs in Nephila clavipes. All prey carried to the hub for storage.

Stage 3. All prey overcome by biting. Post immobilization wrapping occurs at the capture site where it functions as in Stage 2. Additionally, after post imrnobilization wrapping at the capture site prey may be left in situ if the spider already has prey at the hub. Such wrapping acquires a new function: it enables the spider to safely store the prey at the capture site and omit immediate transportation to the hub. This stage is found in some species of Micrathena and Gasteracantha. Some prey stored in the web.

Stage 4. Immobilization biting retained for those prey which can escape rapidly from the web, other prey overcome by immobilization wrapping followed by a short bite. Immobilization wrapping similar in morphology to post immobilization wrapping at the capture site. Function of immobilization wrapping: to increase speed of prey handling, or conversely, to decrease the time spent away from the hub. Post immobilization wrapping at capture site retained for prey overcome by biting (functions: as in 3 , above). Post immobilization wrapping at hub retained for small prey which can be pulled from the web, and also for others which are carried to the hub in the jaws (function: as in 2 above). Some or all of these behaviors shown by Argiope argentata, A. savignyi, A. florida (and probably by other Argiope species), also by Araneus diadematus (Peters I93 I, I933a) and probably many other araneids. Prey stored in web. 
Stage 5. Immobilization biting retained for some prey; other prey overcome by immobilization wrapping. Immobilization wrapping of large prey includes a new behavioral component - in the early stages swathes of silk are thrown over the prey from a distance. The spider may even turn to face away from the prey whilst throwing. Function of new type of throwing: the protection of the spider from the defensive armature of the prey. Found in $\mathrm{Ar}$ giope spp. also Eriophora sp. Prey stored in the web. Figure 2 illustrates the additive nature of this scheme.

\section{Acknowledgements}

The authors are grateful to Drs. M. H. Moynihan and Wm. Eberhard for reading earlier drafts of this paper. The work of Miss H. Mirick was supported by an NSF undergraduate research participation grant.

Eberhard, W.

\section{REFERENCES}

1967. Attack behavior of Diguetid spiders and the origin of prey wrapping in spiders. Psyche 74: 173-181.

Peters, H. M.

1931. Die Fanghandlung der Kreuzspinne (Epeira diademata L.) Experimentelle Analysen des Verhaltens. Z. Vergl. Physiol. 15: 693-748.

1933a. Weitere Untersuchungen über die Fanghandlung der Kreuzspinne (Epeira diademata Cl.), Z. Vergl. Physiol. 19: 47-67.

1933b. Kleine Beiträge zur Biologie der Kreuzspinne Epeira diademata Robinson, M. H. Cl. Z. Morph. Okol. Tiere. 26: 447-468.

1969. Predatory behavior of Argiope argentata (Fabricius). Am. Zool. 9: 161-173. 

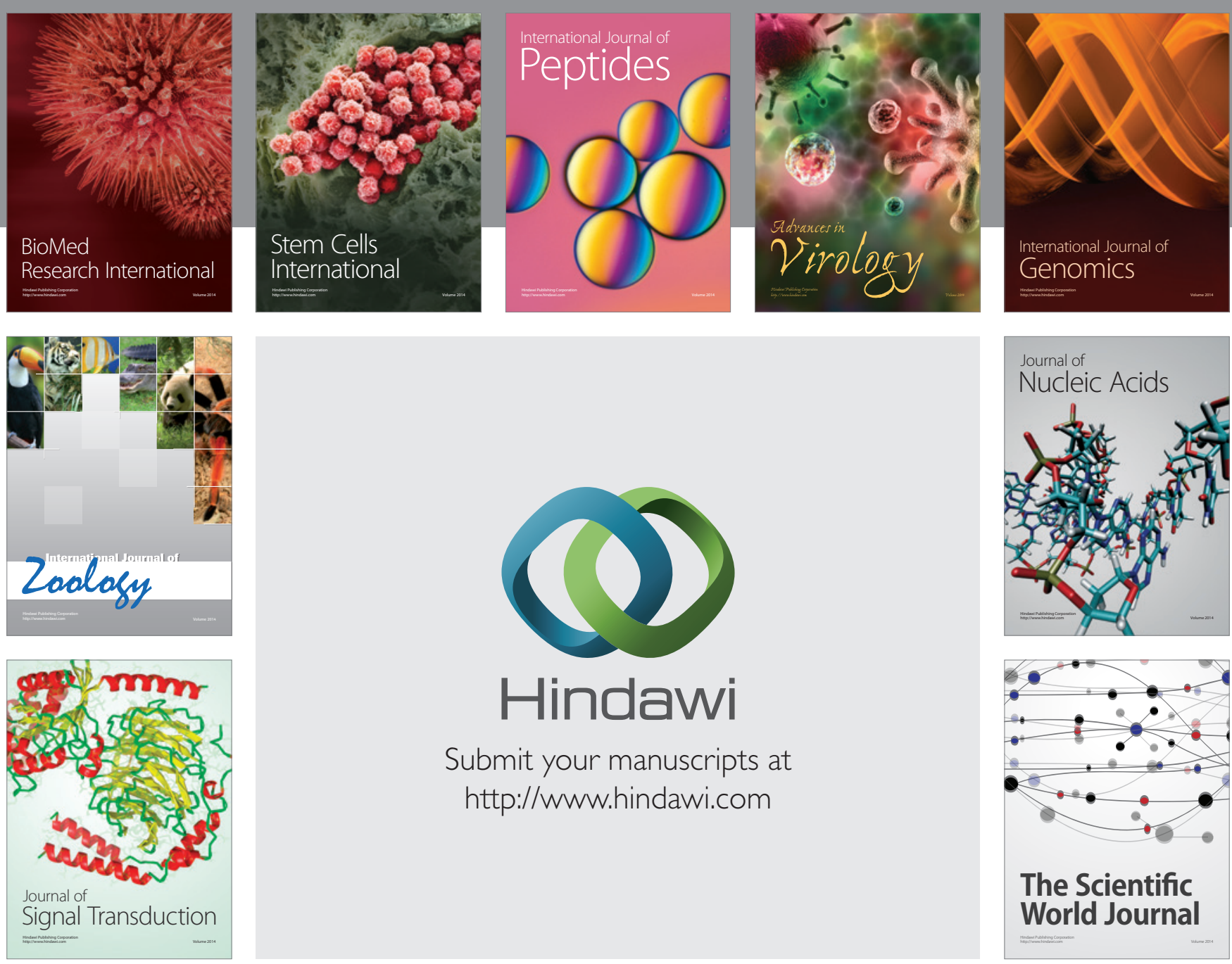

Submit your manuscripts at

http://www.hindawi.com
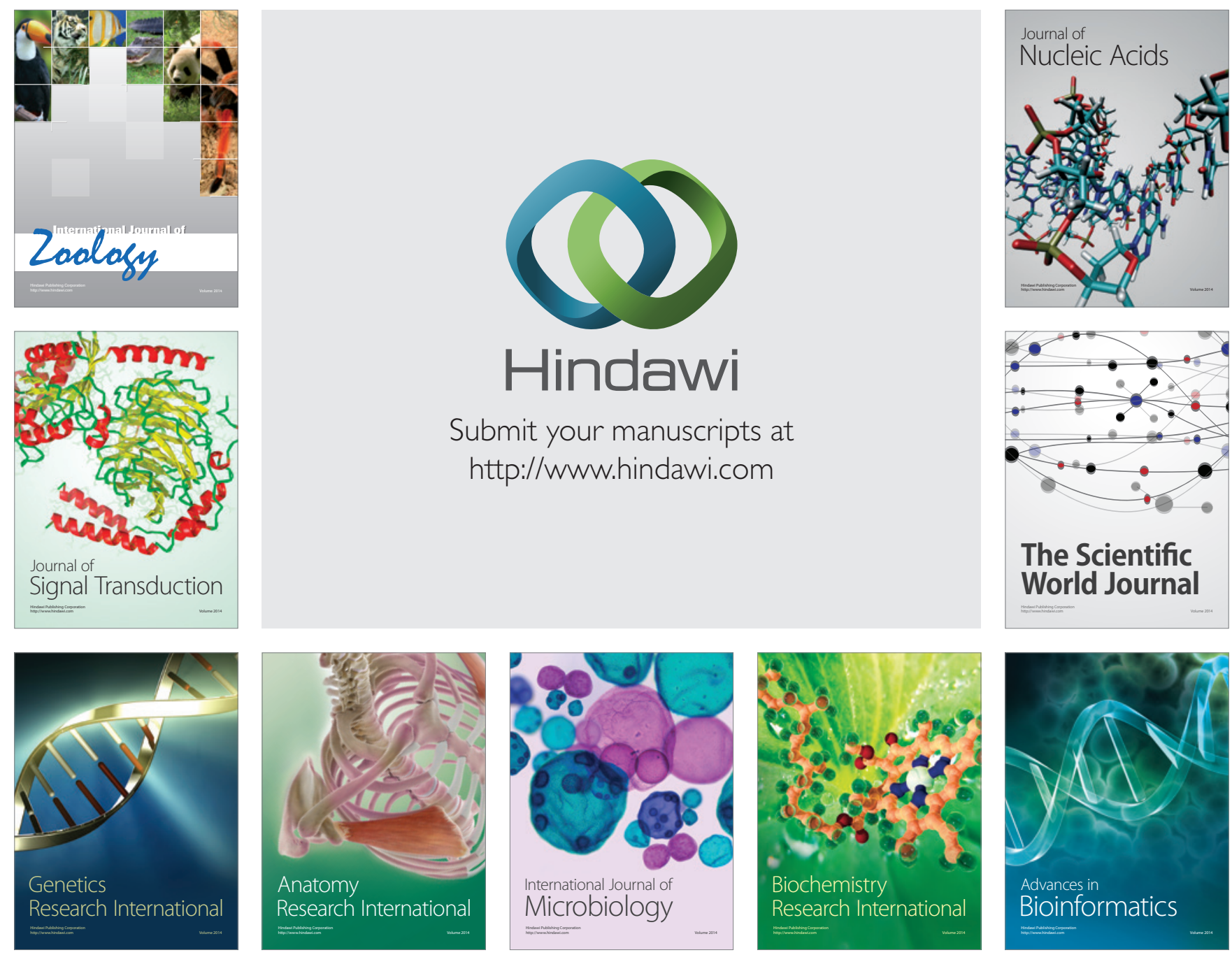

The Scientific World Journal
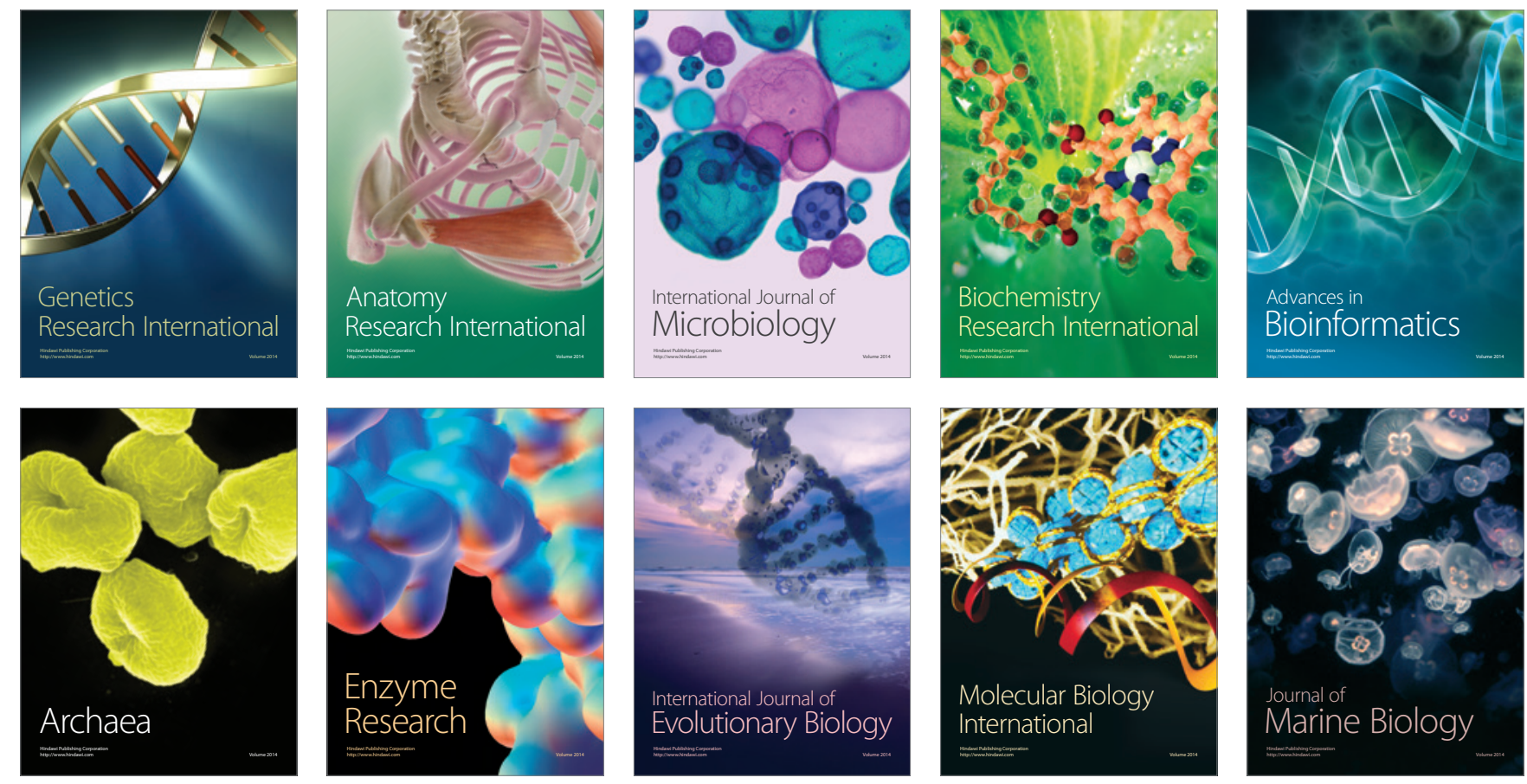